\title{
Salas de Conversa: atividade integrativa de mentoria no contexto da Covid-19
}

\author{
Chat Rooms: integrative mentoring activity in the context of Covid-19
}

\author{
Maria Clara de Sousa Farias Melo' (D) | clarasousa30@gmail.com \\ Laura Ferreira Dias Xavier ${ }^{1}$ (D) lauradxavier27@gmail.com \\ Juliana de Lima Sena' (D) julianasena.ufrn@gmail.com \\ Aurimárcia da Silva Torres ${ }^{1}$ (D) aurimarcia06@gmail.com \\ Francisco Edilson Leite Pinto Junior ${ }^{1}$ (D) edilsonpinto@uol.com.br \\ Maria Bernardete Cordeiro de Sousa' (D) mcbsousa@neuro.ufrn.br
}

\section{RESUMO}

Introdução: O Programa de Mentoria da Universidade Federal do Rio Grande do Norte (UFRN) busca promover o desenvolvimento pessoal e profissional do aluno durante seu período acadêmico. Por meio do método da escuta e do diálogo entre mentores e mentorados, a atividade de mentoria propicia o desenvolvimento da empatia e de outras habilidades emocionais importantes para uma prática médica humanizada e o bom convívio profissional. Busca também trazer para reflexão temas relativos à ética médica, à inserção do médico na comunidade, entre outros. Dentro dessa proposta, no contexto da pandemia do novo coronavírus (Covid-19), o programa passou a desenvolver, além dos encontros on-line regulares, uma atividade integrativa intitulada "Salas de Conversa".

Relato de experiência: Entre junho e dezembro de 2020, realizaram-se, mensalmente, seis salas de conversa com temas diversos. Os encontros ocorreram em salas virtuais e contaram com a participação de todos os grupos de mentoria e mentores cadastrados no programa, além de convidados que estiveram à frente das discussões.

Discussão: O retorno positivo de discentes e docentes mostrou o potencial dessa modalidade de interação de complementar os encontros já desenvolvidos no Programa de Mentoria. A interação virtual apresentou entraves por limitações tecnológicas, todos contornados em tempo hábil, sem prejuízo dessa nova forma de experiência. Conclusão: As Salas de Conversa mostraram-se eficazes na superação das dificuldades de interação impostas pelo distanciamento físico da pandemia de Covid-19. Essa atividade abordou temáticas atuais e pertinentes não apenas à vida do estudante de Medicina, mas também à vida em sociedade, de modo a contribuir tanto para a formação médica quanto para a formação pessoal dos alunos e professores.

Palavras-chave: Mentoria; Educação Médica; Ensino Remoto; Estudante Universitário; Covid-19

\begin{abstract}
Introduction: The Mentoring Program of the Federal University of Rio Grande do Norte seeks to promote the student's personal and professional development during his academic training. Through the method of listening and dialogue between mentors and mentees, the mentoring activity promotes the development of empathy and other emotional skills important for a humanized medical practice and good professional life. It also seeks to bring up themes related to medical ethics, insertion of the doctor in the community, among others. Within this proposal, and against the backdrop of the new coronavirus pandemic, an integrative activity entitled "Chat Rooms" was implemented in addition to the regular online meetings.
\end{abstract}

Experience report: From June to December 2020, six chat rooms with different themes were held monthly. The meetings were held in virtual rooms for all mentoring groups and mentors registered on the program, in addition to guests who were at the forefront of the discussions.

Discussion: The positive feedback from students and mentors showed the potential of this type of interaction to complement the meetings already developed in the mentoring program. The virtual interaction presented obstacles due to technological limitations, all of which were overcome in a timely manner, without detriment to this new form of experience.

Conclusion: The Chat Rooms proved to be effective in resolving the interaction difficulties imposed by the social distancing measures enforced by the Covid-19 pandemic. Bringing current and pertinent themes not only to the medical student's life but to life in the wider society, they contributed both to the medical training and personal training of students and mentors.

Keywords: Mentoring; Medical Education; Remote Education; Covid-19.

${ }^{1}$ Universidade Federal do Rio Grande do Norte, Natal, Rio Grande do Norte, Brasil.

Editora: Patrícia Lacerda Bellodi.

Recebido em 26/02/21; Aceito em 07/04/21.

Avaliado pelo processo de double blind review. 


\section{INTRODUÇÃO}

A origem do uso da palavra mentoria remonta à época da guerra de Troia descrita na Odisseia, de Homero, quando Odisseu confiou seu filho a Mentor, um professor e consultor confiável, atributos presentes quando se busca definir o perfil atual de um mentor ${ }^{1,2}$. Já o conceito atual do que se considera um programa de mentoria foi desenvolvido nos Estados Unidos, na década de 1970, e inserido, nos anos 1990, na área da saúde. A partir daí, cada vez mais escolas de saúde vêm implementando esse tipo de programa em seus cursos, objetivando desde uma melhor interação entre os alunos até o desenvolvimento de habilidades, compartilhamento de experiências e ampliação da rede de relações ${ }^{3}$.

A implementação de programas de mentoria torna-se imprescindível no atual contexto de cursos médicos com índices preocupantes de estresse e esgotamento mental em discentes. Estudos mostram que atividades sociais e benéficas à saúde podem ajudar na identificação mais precoce de alunos mais vulneráveis e na construção de um senso de resiliência de grupo 4 .

Considerando o projeto pedagógico do seu curso de graduação de Medicina, a Universidade Federal do Rio Grande do Norte (UFRN) implementou seu Programa de Mentoria em 2015 e, desde então, constata o sucesso dessa iniciativa a partir de indicadores observados com esse novo componente curricular ${ }^{5}$.

O Programa de Mentoria da UFRN conta com o apoio de um grupo de monitores, denominados mentores juniores, cuja função é organizar e dinamizar os encontros de mentoria com os mentores em relação à logística, tanto na realização das reuniões como na elaboração de calendários da mentoria, propor e discutir temas, convidar os palestrantes para as reuniões, divulgar as reuniões, além de oferecer suporte técnico aos palestrantes. Além disso, o mentor júnior atua como uma ponte entre os alunos e o mentor, buscando manter um padrão de qualidade mínimo entre as reuniões dos diferentes tipos de grupo. Com a implementação das novas mudanças em razão da pandemia da coronavirus disease 2019 (Covid-19), o mentor júnior passou a auxiliar os palestrantes com as novas ferramentas on-line utilizadas e no acompanhamento virtual das reuniões dos grupos de mentoria, atuando como agentes facilitadores da discussão.

As atividades do programa ocorriam de forma presencial até março de 2020 quando, a partir do cenário da pandemia, houve a necessidade de implementação do ensino remoto e adaptações metodológicas para atender a essa nova demanda.

Desse modo, além das reuniões específicas de cada grupo com seus respectivos mentores, o programa iniciou uma nova atividade, na qual todos os grupos da mentoria passaram a integrá-la, sob o título de "Salas de Conversa". Essa atividade integrativa passou a ter uma periodicidade mais frequente, uma vez que era realizada uma única vez, na reunião final nos semestres anteriores. Nessa perspectiva, foi criada uma comissão com cinco mentores e três mentores juniores responsável pela programação e que elaborou um calendário após ouvir sugestões dos grupos da mentoria, selecionando temas pertinentes à formação técnica, humana e ética do profissional médico. Este relato tem por objetivo descrever o desenvolvimento da atividade "Salas de Conversa" e a relação desses temas com os objetivos do Programa de Mentoria, que incluem o desenvolvimento profissional e pessoal dos acadêmicos de Medicina da UFRN.

\section{RELATO DE EXPERIÊNCIA}

O Programa de Mentoria da UFRN, composto por 21 grupos coordenados por 21 mentores e 20 monitores, após a pandemia da Covid-19, precisou reorganizar suas atividades para integralizar a carga horária do primeiro semestre letivo de 2020, acrescentando seis Salas de Conversa aos seis encontros específicos por grupo de mentoria.

Para a realização das Salas de Conversa, a plataforma utilizada para os encontros foi o Google Meet, uma ferramenta integrada ao Google Classroom que permitiu, no máximo, 250 participantes. As reuniões contaram com a participação de oito palestrantes em seis diferentes dias.

\section{Primeira sala de conversa}

A sala de conversa inaugural foi intitulada "Tempos de pandemia: conversa como fonte de saúde", realizada no dia 25 de junho de 2020 e ministrada pela professora convidada Dra. Patrícia Bellodi, então professora da Faculdade de Medicina da Universidade de São Paulo (FMUSP).

O encontro foi realizado em momento crítico mundialmente devido à pandemia de Covid-19 e gerou grande expectativa quanto à sua efetivação para todos que fazem parte do grupo da mentoria, pois as atividades presenciais do curso de Medicina da UFRN haviam sido interrompidas. Essa interrupção ocasionou um impacto muito negativo nos alunos, os quais sofreram com a mudança no curso de suas vidas diante do isolamento social.

Inicialmente, abordaram-se o contexto vivenciado, a ansiedade e a potencial depressão que a situação atual poderia estar gerando nas pessoas e, em especial, nos alunos de Medicina. Foram abordadas diversas maneiras pelas quais uma pandemia poderia afetar e prejudicar a saúde mental dos estudantes. Perguntas como "Com quem temos conversado?" e "Sobre o que temos conversado?" foram guiadoras do debate, bem como "O que fazemos em situações-limite?" e "O que poderíamos fazer para tornar este momento menos traumático?".

A mentoria foi apontada como uma alternativa saudável 
em meio ao caos que estávamos vivenciando, além de tentar focar nossos pensamentos e esforços na nossa saúde.

A importância do bom convívio dentro dos grupos de mentoria também foi citada como uma fonte de suporte social e emocional para os participantes, pois, nos grupos, era possível encontrar apoio para superar as dificuldades de um momento difícil. Esse suporte foi exemplificado com registros de momentos que alguns grupos vivenciaram até mesmo antes de a pandemia acontecer, evidenciando o quão forte pode ser o vínculo que os grupos desenvolvem.

\section{Segunda sala de conversa}

O segundo encontro virtual das Salas de Conversa teve como temática "Boas práticas em telemedicina", realizado no dia 9 de julho de 2020, e foi ministrado pelos professores Dr. José Diniz e Dra. Rosiane Diniz, ambos mentores do programa.

No contexto da pandemia, ficou evidente que a telemedicina foi uma das ferramentas mais utilizadas para que os pacientes não perdessem o seguimento de seus tratamentos e, ao mesmo tempo, não tivessem que correr um risco de uma contaminação potencial da Covid-19 se deslocando até consultórios, unidades de saúde ou hospitais.

A internet foi uma grande aliada para que os pacientes pudessem ter suas necessidades atendidas e não sofressem mais agravos em sua saúde por falta de assistência médica. Consultas feitas por meio de plataformas on-line, contato com médicos e demais profissionais de saúde por meio de aplicativos de mensagens, renovação de receitas e agendamentos de internações e procedimentos foram cada vez mais facilitados e utilizados com o decorrer dos dias de isolamento social.

A nova formatação dos atendimentos que foi adotada por conta da pandemia com certeza deixará seu legado para a prática diária da medicina a posteriori. A atualização dos estudantes e profissionais quanto ao uso das novas tecnologias e também a reflexão sobre o uso ético de tais ferramentas foram apresentadas como essenciais para a não maleficência aos pacientes.

\section{Terceira sala de conversa}

O terceiro momento de discussão teve como tema "Publicidade médica: o que é certo e o que é errado", realizado no dia 23 de julho de 2020 e conduzida pelo médico Dr. Gustavo Xavier, representante do Conselho Regional de Medicina do Estado do Rio Grande do Norte (Cremern).

No encontro, inicialmente houve uma contextualização da recorrência do uso das redes sociais pelos estudantes de Medicina e médicos, que está cada vez mais frequente. As mídias se mostraram como uma ferramenta que pode trazer benefícios, mas o mau uso delas pode ser, muitas vezes, prejudicial diante da lei que protege as boas práticas da profissão médica.
Algumas condutas como a divulgação de dados confidenciais de consultas médicas, exames, procedimentos e imagens de pacientes sem termo de consentimento têm se tornado cada vez mais comuns e podem acarretar danos aos pacientes e aos próprios profissionais de saúde, os quais podem ser impedidos de exercer a medicina.

Os alunos de Medicina também foram colocados como atores nesse cenário, pois muitos são vistos com publicações fora dos padrões de legalidade em suas redes sociais. $O$ alerta para os possíveis danos que tais práticas oferecem foi lançado, e, diante desse contexto, apresentaram-se dicas de como conseguir se manter seguro no ambiente virtual, sem desobedecer às premissas do Código de Ética Médica.

\section{Quarta sala de conversa}

Essa sala de conversa teve como tema "Idade Mídia - por que a pandemia nos levou para 2030?" e foi realizada no dia 25 de setembro de 2020 pelo mentor Dr. Edilson Pinto. Nessa atividade, discutiram-se a era da tecnologia e suas repercussões na sociedade atual, com ênfase no seu impacto dentro da medicina e para o futuro da profissão.

Nessa sala de conversa, contextualizaram-se as novidades dessa nova era tecnológica e todo o seu arsenal, na tentativa de fazer o aluno compreender que não é a instauração de uma era boa ou má, mas cheia de novos elementos que podem ser utilizados para o bem e para o mal.

Também foi abordada a importância que há na constante atualização do profissional médico quanto às ferramentas que surgem para o exercício da medicina. Um médico que se torna capaz de reconhecer e manejar, mesmo que o básico das novas tecnologias em saúde, garante o seu lugar no mercado de trabalho e se torna capaz de oferecer um serviço de melhor qualidade para o seu paciente.

Não obstante a isso, as novas tecnologias podem não representar uma ameaça para o profissional médico. Muito se fala que algumas especialidades correm o risco de ser substituídas pelas máquinas, mas a relação médico-paciente e a empatia para com as pessoas que, muitas vezes, se encontram em seus momentos mais críticos da vida são fatores insubstituíveis na prática diária da medicina.

\section{Quinta sala de conversa}

A temática da quinta sala de conversa foi "Carreira, mercado e planejamento profissional", realizada no dia 29 de outubro de 2020 pelo mentor Dr. Francisco Irochima Pinheiro. A palestra deu-se de maneira bem dinâmica, pois o debatedor instigava os alunos sobre determinado tema respondendo às perguntas que imediatamente geravam novos questionamentos. 
A criatividade aliada ao empreendedorismo social e sustentável perpassava cada rodada de conversas, em que até o desafio da "caixa de fósforo" ("Como manter uma vela acesa e em pé, sem ela tocar no chão, tendo apenas uma caixa de fósforo, uma vela e várias tachinhas para madeira?") foi abordado. Ao aguçar a criatividade dos alunos, o debatedor estava tentando despertar os talentos e as aptidões naturais que deveriam ser estimulados ao longo de cada disciplina do curso médico, inclusive terminou com uma sugestão desafiadora da criação de novas disciplinas optativas no curso médico para atender aos desafios educacionais contemporâneos, tais como: design digital, gestão de redes digitais voltadas para educação, entre outros.

\section{Sexta sala de conversa}

A sexta sala de conversa teve como tema "Racismo científico e relações étnico-raciais" e foi realizada no dia 27 de novembro de 2020 pelos convidados Dra. Angela Facundo Navia (professora) e Dr. Luiz Meza Álvares (pós-doutorando) do Departamento de Antropologia da UFRN.

Na apresentação das raízes históricas do racismo, dentro do próprio ambiente científico, os convidados utilizaram as taxonomias com explicações baseadas em características biológicas sem considerar os contextos sócio-históricos, próprios do lluminismo, para hierarquizar grupos de humanos e justificar a dominação, dentro do contexto da expansão colonial europeia. Foi atualizada essa visão, na qual o conceito de raça já não é mais aceito, mas o racismo continua.

$O$ racismo científico teve repercussões no Brasil e estava no seu auge no final do século XIX. A ausência de políticas públicas no Brasil colonial após a abolição gerou uma desigualdade ainda hoje presente. Esses indicadores refletem a existência do racismo estrutural: no mercado de trabalho, a desocupação é três vezes maior para os negros; nos setores da economia, os negros são maioria na agricultura, na construção civil e nos serviços domésticos; e o salário médio deles é cerca de $50 \%$ menor, com igual nível de formação (ensino superior) ${ }^{7,8}$.

Abordaram-se aspectos relacionados às leis específicas aprovadas no Brasil contra o racismo e às políticas de cotas no ensino superior e nos concursos públicos federais, além de outras políticas afirmativas necessárias, como a de transferência de renda.

Ao final da palestra, os alunos puderam fazer perguntas ou comentários tanto por meio da voz, com a abertura dos microfones, como por meio da escrita no chat. As dúvidas ou os comentários eram lidos ao palestrante por algum monitor, e, assim, muitos discentes relataram suas vivências dentro do curso. Uma aluna em especial relatou comentários de professores com conotação racial, assim como posições de colegas que se colocam como "neutros" afirmando "não existir racismo no Brasil" ou sem a devida compreensão do que seria a questão social relacionada ao racismo, ao afirmarem "Sou branco e já sofri preconceito racial". Essa foi uma das salas de conversa com maior discussão e interação, possivelmente por todo o contexto envolvido no tema.

\section{DISCUSSÃO}

Levando-se em consideração o feedback dos alunos e dos monitores por meio dos chats nas reuniões e de comentários realizados em redes sociais após participarem das salas de conversa, pôde-se perceber que essa modalidade foi capaz de proporcionar uma boa interação entre os discentes, e entre discentes e docentes, o que foi avaliado positivamente, sobretudo diante do cenário de isolamento social vivenciado. Essa relação foi efetuada primordialmente por meio do chat, no qual se postavam comentários e dúvidas acerca do tema abordado.

Foi verificado um alto índice de interesse e de satisfação, justificado pelo grande número de participantes, pelo significativo engajamento no chat e pelos elogios realizados às Salas de Conversa, como também pelo aumento de interações entre mentores e alunos evidenciado nos grupos de redes sociais. Tais interações se tornam essenciais no contexto de Covid-19 e isolamento social. Estudos mostram que a solidão causada pelo isolamento social afeta o corpo humano em três esferas principais, sendo elas a fisiológica, a psicológica e a comportamental, com impacto significativo no sistema cardiovascular e imune, inclusive diminuindo a resistência às doenças eàs infecções ${ }^{9}$. Há indícios de que o uso da comunicação social virtual, principalmente aquela por meio de vídeo, tenha grande importância no enfrentamento dessa solidão causada pelo isolamento social. Assim, é fundamental incentivar a continuidade de discussão on-line, independentemente do tema, de forma que um contato social seja mantido, sendo importante incentivar a interação e visualização por vídeo de todos os participantes ${ }^{10}$.

As Salas de Conversa apresentaram temáticas relevantes para a formação médica, como as de "Boas práticas em telemedicina", "Publicidade médica: o que é certo e o que é errado" e "Carreira, mercado e planejamento profissional". Foram também capazes de contribuir com temas estruturantes, como "Racismo científico e relações étnico-raciais", e com relação ao momento de pandemia, com os temas "Tempos de pandemia: conversa como fonte de saúde" e "Idade Mídia - por que a pandemia nos levou para 2030?".

O tema do racismo, por exemplo, é de essencial discussão, afinal, no ano de 2020, houve inúmeras mortes de homens e mulheres negros em diferentes países. Os centros acadêmicos da área das ciências da saúde, por muito tempo, permaneceram em silêncio sobre o racismo. Entretanto, na 
atualidade, urge que as escolas médicas se posicionem e trabalhem contra o racismo. Na medicina, muitos insistem em discutir apenas a esfera biológica, mas os relatos trazidos pelos alunos na sala da conversa demonstram que é necessária uma nova abordagem dentro do curso médico ${ }^{11}$.

Ainda contextualizando a pertinência dos temas, um estudo nas escolas médicas coreanas mostra uma crescente necessidade dos acadêmicos por orientação profissional. Assim como no Brasil, lá os alunos ingressam no curso muitas vezes sem clareza do que desejam na carreira ou o que esperam do curso. Nesse sentido, a discussão sobre a carreira médica e a inserção profissional, a telemedicina e a publicidade médica é essencial para gerar no aluno a reflexão acerca do que ele deseja na carreira e o que deve buscar para uma melhor inserção profissional, de modo que haja médicos que sabem o que querem e não uma manada de perdidos na profissão ${ }^{12}$.

No tocante a problemas técnicos, em alguns encontros, a quantidade máxima de pessoas (250) na sala virtual foi atingida, não tendo sido possível atender todos os alunos matriculados (um total de 317) no referido período. Não houve intercorrências relacionadas à conexão e qualidade da internet.

O educador Ken Robinson ${ }^{13}$, em seu livro Libertando o poder criativo, mostra o perigo de as universidades se voltarem apenas para o conhecimento com fim específico e para o raciocínio lógico-dedutivo - em que currículos fomentam apenas habilidades associadas ao hemisfério esquerdo do cérebro. Isso seria o mesmo que "treinar alguém para uma corrida exercitando apenas uma perna, deixando que os músculos da outra atrofiassem"13.

Essas salas de conversas fizeram "verdadeiras ginásticas" no nosso cérebro e nas nossas almas, pois tiveram a capacidade de nos manter ao mesmo tempo "longe dos olhos, mas perto do coração".

\section{CONCLUSÃO}

Diante dos tópicos abordados, é possível concluir que as Salas de Conversa foram eficazes como uma nova ferramenta desenvolvida dentro do Programa de Mentoria da UFRN, quando este buscou por adequações metodológicas, durante o período da pandemia de Covid-19. A ocorrência da aproximação dos grupos de mentoria em um espaço virtual comum contribuiu de forma significativa para a troca de experiências e para o fortalecimento dos laços entre mentores e discentes em um momento de distanciamento físico. Ademais, essa atividade trouxe à discussão temas pouco debatidos nas grades curriculares universitárias e que acrescentaram qualidade à formação ética e humanizada de profissionais da saúde. Vislumbra-se, assim, a permanência das Salas de Conversa integradas às dinâmicas do Programa de Mentoria, tanto por haver proporcionado uma maior integração entre os atores da mentoria quanto pela inovação dos temas selecionados.

\section{CONTRIBUIÇÃO DOS AUTORES}

Os autores contribuíram de forma igualitária para a produção do artigo e as atividades relatadas.

\section{CONFLITO DE INTERESSES}

Declaramos não haver conflito de interesses.

\section{FINANCIAMENTO}

Declaramos não haver financiamento.

\section{REFERÊNCIAS}

1. Ortega G, Smith C, Pichardo MS, Ramirez A, Soto-Greene M, Sánchez JP. Preparing for an academic career: the significance of mentoring. MedEdPORTAL. 2018;14:10690.

2. Chopra V. Will you be my mentor? Four archetypes to help mentees succeed in academic medicine. JAMA Intern Med. 2017;27:E1-E2.

3. Martins AF, Bellodi PL. Mentoring: uma vivência de humanização e desenvolvimento no curso médico. Interface Comun Saúde Educ 2016;20(58):715-26.

4. Silva V, Costa P, Pereira I, Faria R, Salgueira AP, Costa MJ, et al. Depression in medical students: insights from a longitudinal study. BMC Med Educ. 2017;17(1):1-9.

5. Moreira SNT, de Albuquerque ICS, Pinto Junior FEL, Gomes AHB. Programa de Mentoria do curso de Medicina da Universidade Federal do Rio Grande do Norte: atividades integrativas em foco. Rev Bras Educ Med. 2020;44(4) e169.

6. Conselho Federal de Medicina. Código de Ética Médica: Resolução nº 2.217, de 27 de setembro de 2018, modificada pelas Resoluções CFM no 2.222/2018 e 2.226/2019. Brasília: CFM; 2019.

7. Instituto Brasileiro de Geografia e Estatística. Desigualdades sociais por cor ou raça no Brasil. Rio de Janeiro: IBGE; 2019 [acesso em 24 fev 2021] Disponível em: https://biblioteca.ibge.gov.br/index.php/biblioteca-catalo go?view=detalhes\&id=2101681.

8. Ribeiro $B C$, Komatsu BK, Menezes-Filho. Diferenciais salariais por raça e gênero para formados em escolas públicas ou privadas [Policy Paper n 45]. São Paulo: Insper; 2020 [acesso em 24 fev 2021]. Disponível em: https:// www.insper.edu.br/wp-content/uploads/2020/07/Policy-Paper-45.pdf.

9. D'Acquisto F, Hamilton A. Cardiovascular and immunological implications of social distancing in the context of Covid-19. Cardiovasc Res. 2020;116(10):129-31.

10. Bzdok D, Dunbar RIM. The neurobiology of social distance. Trends Cogn Sci. 2020;24(9):717-33.

11. Sharma $M$, Kuper $A$. The elephant in the room: talking race in medical education. Adv Health Sci Educ Theory Pract. 2017;22(3):761-4.

12. Hur Y, Cho AR, Kwon M. Development of a systematic career coaching program for medical students. Korean J Med Educ. 2018;30(1):41-50.

13. Robinson K. Libertando o poder criativo: a chave para o crescimento pessoal e das organizações. São Paulo: HSM, 2012, p. 120. 